\title{
First European guidelines on syndromic surveillance in human and animal health published
}

A Hulth (anette.hulth@folkhalsomyndigheten.se)

1. Public Health Agency of Sweden, Stockholm, Sweden

Citation style for this article:

Hulth A. First European guidelines on syndromic surveillance in human and animal health published. Euro Surveill. 2014;19(41):pii=20927. Available online: http:// www.eurosurveillance.org/ViewArticle.aspx?Articleld=20927

On 11 October 2014, the first European guidelines on syndromic surveillance in human and animal health, the 'Triple-S guidelines for designing and implementing a syndromic surveillance system', were published [1].The guidelines are one of the main outcomes of the European Union (EU) -funded project 'Triple-S', which main aim has been to increase the European capacity for near-real time surveillance and monitoring of health-related events in the animal and the human health sectors.

The Triple-S guidelines provide evidence-based recommendations and suggestions for each step of the setup, use and assessment of a syndromic surveillance system. They aim to encourage a common understanding of the structure and utility of systems, and improve communication among European countries on critical public health threats. The guidelines are designed to be useful in the context of the wide range of health systems and data sources found in European countries, and their main principles can be applied globally. The guidelines are intended for public health professionals and epidemiologists working in human or animal health surveillance who would like to use syndromic surveillance to support existing surveillance systems and public health monitoring.

Triple-S was a project co-financed by the EU through the Executive Agency for Health and Consumers. It started in September 2010 and formally ended in December 2013, and was coordinated by the French Institute for Public Health Surveillance (InVS) in Paris. It involved twenty four organisations from thirteen European countries. As an outcome of the first meeting, held in November 2010, an updated definition of 'syndromic surveillance' was published in 2011 [2].

All publications originating from the Triple-S project, including the guidelines and reports on conducted inventories and site visits, can be found on the Triple-S web site (www.syndromicsurveillance.eu).
References

1. Triple-S. The syndromic surveillance project. Triple-S; Oct 2014. Available from: http://www.syndromicsurveillance.eu/

2. 2. Triple $S$ Project. Assessment of syndromic surveillance in Europe. Lancet, 2011;378(9806):1833-4. 